Pacific Journal of Mathematics

INTERIOR VARIATIONS AND SOME EXTREMAL PROBLEMS
FOR CERTAIN CLASSES OF UNIVALENT FUNCTIONS 


\title{
INTERIOR VARIATIONS AND SOME EXTREMAL \\ PROBLEMS FOR CERTAIN CLASSES OF \\ UNIVALENT FUNCTIONS
}

\author{
VIKRAMADITYA Singh
}

1. Introduction. The theory of regular univalent functions in the unit circle $U_{z}$ has been developed for various subclasses, for example, the class of real univalent functions which leads to symmetric domains, the class of bounded univalent functions whose image domain lies with in the unit circle and the functions for which the image domains are convex or star-like. The approach through the calculus of variations has been used very successfully towards the solution of extremal problems belonging to the various classes and also towards the determination of the extremal domains. The purpose of the present paper is to show how the method of interior variations due to Schiffer [1] can be adapted for the following subclasses:

(i) The class $V$ of symmetric regular univalent-functions $f(z)$ in $U_{z}$ which have the form $f(z)=z+\sum_{n=2}^{\infty} a_{n} z^{n}$ with real $a_{n}$. In particular we show that if $\varphi\left(a_{2}, a_{3}, \cdots, a_{n} ; \bar{a}_{2}, \bar{a}_{3}, \cdots, \bar{a}_{n}\right)$ is a real valued function which is symmetric and analytic in $a_{\nu}$ and $\bar{a}_{\nu}(\nu=2,3, \cdots, n)$ and where $\left\{a_{n}\right\}$ are the coefficients in the power series expansion of the more general class $V_{1}$ of regular univalent functions then, under the assumption that the function $f(z)$ whose coefficients $\left\{a_{\nu}\right\}$ maximize $\varphi\left(a_{2}, \cdots, a_{n}\right.$, $\left.\bar{a}_{2}, \cdots, \bar{a}_{n}\right)$ is symmetric, the functional differential equation satisfied by $f(z)$ in the general class $V_{1}$ is the same as the functional differential equation satisfied by $f(z)$ in the class $V$.

(ii) The class $S$ of bounded univalent functions $f(z)$ in $U_{z}$ which are normalized so that $f(0)=0,|f(z)| \leqq 1$ and at a fixed point $\zeta \in U_{z}$, $f(\zeta)=\omega$. In particular we find the functions which maximize or minimize $\left|f^{\prime}(\zeta)\right|$.

(iii) The class $\Sigma$ of bounded univalent functions $f(z)$ in $U_{z}$ which are real on the real axis and are normalized so that $f(0)=0, \quad|f(z)| \leqq 1$ and at a fixed point $\zeta$ on the real axis $f(\zeta)=\omega$. In particular we find the functions which maximize or minimize $f(\eta)$ for real $\eta \in U_{z}$.

We observe that the existence and uniqueness of the solutions of these problems is assured because the families of functions belonging to the classes $V, S$ and $\Sigma$ are normal and compact.

2. Real univalent functions. Let $D$ be the image in the $W$-plane

Received October 15, 1956, Prepared under Contract Nonr-225 (11) (NR-041-086) Office of Naval Research. 
$W=f(z) \in V$ of $|z| \leqq 1$ and let us consider the Schiffer variation

$$
W_{1}^{k}=W+a \rho^{2} \underset{W_{0}\left(W-W_{0}\right)}{W}+\bar{a} \rho^{2} \quad \begin{aligned}
& W \\
& W_{0}\left(W-W_{0}\right)
\end{aligned},
$$

where $\bar{W}_{0}$ is an interior point of $D$. It is easily seen that for small enough $\rho$ say $\rho_{1}<\rho,\left|W-W_{0}\right|=\rho_{1}$ and $\left|W-\bar{W}_{0}\right|=\rho_{1}$ lie entirely in $D$ and $W_{1}^{*}$ is univalent on the boundary $C$ of $D$ and maps it univalently on to the boundary $C^{*}$ of the new domain $D^{*}$. Further, we see that $W_{1}^{*}=0$ for $W=0$ and that $W_{1}^{*}$ is real for real values of $W$. Thus if $W$ is a symmetric univalent function which vanishes at the origin we have obtained another neighbouring function which also has the same properties. In order to be able to add some side conditions to the function $W$ we consider the variation

$$
W^{*}=W+\rho^{2} \sum_{\nu=1}^{p}\left\{\frac{\alpha_{\nu} W}{\left(W-W_{\nu}\right) W_{\nu}}+\begin{array}{c}
\bar{\alpha}_{\nu} W \\
\left(W-W_{\nu}\right) W_{\nu}
\end{array}\right\},
$$

where $p$ is an integer $\geq 1$. This variation is of the same type as (1) and has the independent constants $\left(\alpha_{\nu}\right)^{\prime}$ which can be used to satisfy the side conditions, if any.

The technique of getting the variation formula for $f(z)$ under the variation (2) is similar to that used in [2] in getting the variation formula for $f(z)$ under the variation $W^{*}=W+a \rho^{2} /\left(W-W_{0}\right)$. For the sake of completeness we mention that we first find the variation formula for the Green's function $G(W, 0)$ of $D$ under the variation (2). We thus have [3]

$$
\delta G(W, 0)=\Re\left[\begin{array}{c}
\rho^{2} \\
2 \pi i
\end{array} \int_{\Gamma} p^{\prime}(\eta, 0) p^{\prime}(\eta, W) \varphi(\eta) d \eta\right]+O\left(\rho^{3}\right),
$$

where

$$
\varphi(W)=\sum_{\nu=1}^{p}\left\{\begin{array}{c}
\alpha_{\nu} W \\
\left(W-W_{\nu}\right) W_{\nu}
\end{array}+\begin{array}{c}
\bar{\alpha}_{\nu} W \\
\left(W-W_{\nu}\right) W_{\nu}
\end{array}\right\},
$$

and $p(W, \eta)$ is the analytic function whose real part is the Green's function $G(W, \eta)$ and $\Gamma$ is a curve system in $D$ which is homotopic to $C$ and such that $\varphi(W)$ is analytic in the ring system bounded by $C$ and $\Gamma$. If now $z=\varphi(W)$ is the inverse function of $W=f(z)$ then the relationship of the Green's function $G(W, \omega)$ to the function $\varphi(W)$ is given by

$$
G(W, \omega)=-\log \left|\begin{array}{c}
\varphi(W)-\varphi(\omega) \\
1-\varphi(\omega) \varphi(W)
\end{array}\right|
$$

and in particular 


$$
G(W, 0)=\log \frac{1}{|\varphi(W)|} .
$$

Proceeding in this way we find that the variation formula for $f(z)$ is given by

$$
\begin{aligned}
& f^{*}(z)=f(z)+\sum_{\nu=1}^{p} \alpha_{\nu} \rho^{2}\left[\begin{array}{cc}
z^{2} & f^{\prime}(z) \\
1-z t_{\nu} & t_{\nu} f^{\prime \prime 2}\left(t_{\nu}\right)
\end{array}+\frac{z f^{\prime}(z)}{z_{\nu} f^{\prime 2}\left(z_{\nu}\right)} \quad \frac{1}{z_{\nu}-z}\right. \\
& \left.+\frac{f(z)}{f\left(z_{\nu}\right)\left(f(z)-f\left(z_{\nu}\right)\right)}\right]+\Sigma \bar{\alpha}_{\nu} \rho^{2}\left[\frac{z^{2}}{1-z \bar{z}_{\nu},} \frac{f^{\prime}(z)}{\bar{z}_{\nu} f^{\prime \prime 2}\left(z_{\nu}\right)}+\frac{z f^{\prime}(z)}{t_{\nu} f^{\prime 2}\left(t^{\nu}\right)} \frac{1}{t_{\nu}-z}\right. \\
& \left.+\frac{f(z)}{f\left(t_{\nu}\right)\left(f(z)-f\left(t_{\nu}\right)\right)}\right]+O\left(\rho^{3}\right),
\end{aligned}
$$

where

$$
t_{\nu}=\varphi\left(\bar{W}_{\nu}\right) \text { or } \quad f\left(t_{\nu}\right)=f\left(\bar{z}_{\nu}\right) \text {. }
$$

Let $f(z)$ have the following power series expansion

$$
f(z)=z+\sum_{n=2}^{\infty} a_{n} z^{n}
$$

where the $a_{n}$ are real. Then denoting by $a_{n}^{*}$ the coefficient of $z^{n}$ in $f^{*}(z)$ and substituting these expansions in (6) and equating the coefficients of $z^{n}$ on both sides we get

$$
\begin{aligned}
a_{n}^{*}= & a_{n}+2 \Re\left\{\rho ^ { * } \sum _ { \nu = 1 } ^ { n } \alpha _ { \nu } \left[A _ { \nu } \sum _ { m = 1 } ^ { n - 1 } \left(z_{\nu}^{m}+z_{\nu}^{-m}(n-m) a_{n-m}+n_{a n}+z_{\nu}^{n-1}\right.\right.\right. \\
& \left.\left.+z_{\nu}^{-(n-1)}+T_{n}\left(f\left(z_{\nu}\right)\right)\right]\right\}+O\left(\rho^{3}\right),
\end{aligned}
$$

where

$$
A_{\nu}=\frac{1}{z_{\nu}^{2} f^{\prime \prime}\left(z_{\nu}\right)}
$$

and $T_{n}(f(z))$ are given by the formula

$$
\frac{f(z)}{f\left(t_{\nu}\right)\left(f(z)-f\left(t_{\nu}\right)\right)}=\sum_{n=1}^{\infty} z^{n} T_{n}\left(f\left(t_{\nu}\right)\right) .
$$

We remark that (8) is the variation formula for the coefficient $a_{n}$ given by (7) and for $\nu=1$ it agrees with the variation formula obtained by Schiffer [2] for $\left|a_{n}\right|$ when $a_{n}$ are complex. Further, if we put $\delta a_{n}$ $=a_{n}^{*} / a_{1}^{*}-a_{n}$ then $d a_{n}=\delta a_{n}+O\left(\rho^{3}\right)$ and we find that $\delta a_{n}$ in the present case is twice $\delta \alpha_{n}$ in the general case $a_{n}=\alpha_{n}+i \beta_{n}$. 
Now let us consider a function $\varphi\left(a_{2}, \cdots, a_{n}, \bar{a}_{2}, \cdots, \bar{a}_{n}\right)$ which is symmetric and analytic in $\alpha_{\nu}$ and $\bar{a}_{\nu}$ and has real coefficients. Then, because $a_{\nu}=\alpha_{\nu}+i \beta_{\nu}$, we can write $F\left(\alpha_{2}, \cdots, \alpha_{n} ; \beta_{2}, \cdots, \beta_{n}\right)=\varphi\left(a_{2}, \cdots, a_{n}\right.$; $\left.\bar{a}_{2}, \cdots, \bar{a}_{n}\right)$, and the function $F$ will contain only even powers of $\beta_{\nu}$ 's and

$$
\frac{\partial F}{\partial \beta_{\nu}}=0 \text { if } \beta_{2}=\beta_{3}=\cdots=\beta_{n}=0 .
$$

Further, the condition for the extremum of $F$ in the general case when $a_{\nu}$ are complex is

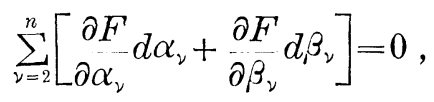

which in the limit when $\rho \rightarrow 0$ can be written as

$$
\sum_{\nu=1}^{n}\left[\frac{\partial F}{\partial \alpha_{\nu}} \delta \alpha_{\nu}+\frac{\partial F}{\partial \beta_{\nu}} \delta \beta_{\nu}\right]=0 .
$$

If $a_{\nu}$ are real then in view of (10), (11) reduces to

$$
\sum_{\nu=2}^{n} \frac{\partial F}{\partial \alpha_{\nu}} \delta \alpha_{\nu}=0
$$

We will obtain the same equation if we look for the solution of the extremumproblem in the particular class $V$ of real functions. Thus under the assumption that the extremum function is real the functional differential equation in the general case will coincide with the differential equation in the symmetric case. We know from compactness arguments that the problem $\varphi=\max$ has a solution in $V$. We also know that the same problem has a solution in the general class $V_{1}$. What we have shown is that both extremum functions satisfy the same functional differential equation. This implies that either there are many solutions of the problem in the class $V_{1}$ or that the solution lies in the class $V$. Thus in particular the coefficient problem $a_{n} \bar{a}_{n}=\max$ leads to the same functional differential equation for real univalent functions as for the general class.

3. Bounded univalent functions. We now consider a variation which transforms a function of the class $S$ into another function of the class $S$. We will first obtain a variation which keeps the origin fixed and also keeps the unit circumference fixed. We will then add the side condition that for a fixed $\zeta, f(\zeta)=\omega$. Let 


$$
W_{3}^{*}=W+a \rho^{2} \underset{W-W_{0}}{W}-\stackrel{\bar{a} \rho^{2} W^{2}}{1-W_{0} W}+O\left(\rho^{4}\right),
$$

where $O\left(\rho^{4}\right)$ can be suitably chosen and $W_{0}$ is an interior point of the image domain of $|z| \leqq 1$ by $f(z)$. For small enough $\rho, W_{2}^{*}$ is a univalent function of $W$ outside $\left|W-W_{0}\right|=\rho_{1}<\rho$ such that $W_{2}^{*}=0$ for $W=0$ and keeps the unit circumference $|W|=1$ fixed. In fact, to prove the latter, we observe that when

$$
|W|=1, \quad W=\frac{1}{W}
$$

and

$$
\left|W_{2}^{*}\right|=\left[1+4 \rho^{4}\left\{\Im\left(\frac{a}{W-W_{0}}\right)\right\}^{2}\right]^{1 / 2}+O\left(\rho^{4}\right)
$$

So to the order $\rho^{2}$ the unit circumference is kept fixed. By adding to (13) term of the order of $\rho^{4}$ one could make the unit circumference fixed. To see this let us denote by $D^{*}$ the boundary of the domain to which $W_{2}^{*}$ maps the unit circumference $|W|=1$. Now by the Riemann mapping theorem there exists an analytic function $S\left(W_{2}^{*}\right)$ which vanishes at the origin and maps $D^{*}$ univalently on the unit circumference $\left[S\left(W_{2}^{*}\right) \mid\right.$ $=1, W_{2}^{*} \in D^{*}$. From the boundary behavior (14) of $W_{2}^{*}$ when $W_{2}^{*} \in D^{*}$ we then conclude that

$$
\left|\begin{array}{c}
S\left(W_{2}^{*}\right) \\
W_{2}^{*}
\end{array}\right| \simeq 1+O\left(\rho^{4}\right)
$$

Since $\log \left|S\left(W_{2}^{*}\right) / W_{2}^{*}\right|$ is harmonic in $\left|W_{2}^{*}\right|<1$, we conclude, by the maximum principle that (15) holds everywhere inside the unit circle. Thus

$$
S\left(W_{2}^{*}\right)=S^{*}(W)=W_{2}^{*}+O\left(\rho^{4}\right)
$$

for $|W|=1$. We have thus obtained a function $S^{*}(W)$ which maps the unit circumference $|W|=1$ onto itself and differs from $W_{2}^{*}$ by $O\left(\rho^{4}\right)$.

A more general type of variation which can take care of some extra side conditions can be written in the form

$$
W^{*}=W+\sum_{\nu=1}^{n}\left[\begin{array}{c}
a_{\nu} \rho^{2} W \\
W-W_{\nu}-\frac{\bar{a}_{\nu} \rho^{2} W^{2}}{1-W_{\nu} W}
\end{array}\right]+O\left(\rho^{4}\right) .
$$

Taking $n=1$ we get according to the procedure outlined in $\S 2$ the following variation formula

$$
\varphi^{*}(W)=\varphi(W)-a_{\mathrm{v}} \rho^{2} A\left(W, W_{0}\right)+\bar{a}_{\mathrm{n}} \rho^{2} B\left(W, \bar{W}_{0}\right)
$$




$$
-a_{1} \rho^{3} A\left(W, W_{1}\right)+\vec{a}_{1} \rho^{2} B\left(W, \bar{W}_{1}\right)+O\left(\rho^{1}\right)
$$

where

$$
A\left(W, W_{0}\right)=\frac{W \varphi^{\prime}(W)}{W-W_{0}}+\frac{W_{0} \varphi^{\prime 2}\left(W_{0}\right) \varphi(W)}{\varphi\left(W_{0}\right)\left(\varphi\left(W_{0}\right)-\varphi(W)\right)},
$$

and

$$
B\left(W, \bar{W}_{0}\right)=\frac{W^{2} \varphi^{\prime}(W)}{1-W W_{0}}-\frac{\varphi^{2}(W) \bar{W}_{0} \varphi^{\prime 2}\left(W_{0}\right)}{\varphi\left(W_{0}\right)\left(1-\varphi(W) \varphi\left(W_{0}\right)\right)} .
$$

Since we require that for all $f(z) \in S$ and fixed $\zeta, f(\zeta)=\omega$, we must have $\varphi^{*}(\omega)=\varphi(\omega)=\zeta$. Thus we obtain the determining relation between $a_{0}$ and $a_{1}$

$$
-a_{0} A\left(\omega, W_{0}\right)+\bar{a}_{0} B\left(\omega, \bar{W}_{0}\right)-a_{1} A\left(\omega, W_{1}\right)+\bar{a}_{1} B\left(\omega, \bar{W}_{1}\right)+O\left(\rho^{2}\right)=0 .
$$

We shall see that in general we can prescribe $W_{0}, W_{1}$ and $a_{0}$ arbitrarily and adjust $a_{1}$ such that (20) holds.

Again, as $\varphi^{\prime}(W)=1 / f^{\prime}(z)$ we see that the minimum and maximum of $\left|f^{\prime}(\zeta)\right|$ would be given by the maximum and minimum of $\left|\varphi^{\prime}(\omega)\right|$. Thus the necessary condition for the extremum of $\left|f^{\prime}(\zeta)\right|$ is

$$
\begin{aligned}
& \Re\left[a_{0} \frac{A^{\prime}\left(\omega, W_{0}\right)}{\varphi^{\prime}(\omega)}-\bar{a}_{0} \frac{B^{\prime}\left(\omega, \bar{W}_{0}\right)}{\varphi^{\prime}(\omega)}+a_{1} \frac{A^{\prime}\left(\omega, W_{1}\right)}{\varphi^{\prime}(\omega)}-\bar{a}_{1} \frac{B^{\prime}\left(\omega, \bar{W}_{1}\right)}{\varphi^{\prime}(\omega)}\right] \\
&+O\left(\rho^{2}\right)=0,
\end{aligned}
$$

where $A^{\prime}\left(\omega, W_{0}\right)$ and $B^{\prime}\left(\omega, \bar{W}_{0}\right)$ are, respectively, the derivatives of $A\left(\omega, W_{0}\right)$ and $B\left(\omega, \bar{W}_{0}\right)$ with respect to the first argument.

The extremum condition (21) can also be written in the form

$$
a_{\lrcorner} C\left(\omega, W_{0}\right)-\bar{a}_{0} \overline{C\left(\omega, W_{0}\right)}+a_{1} C\left(\omega, W_{1}\right)-\bar{a}_{1} \overline{C\left(\omega, W_{1}\right)}+O\left(\rho^{2}\right)=0,
$$

where

$$
C\left(\omega, W_{0}\right)=\frac{A^{\prime}\left(\omega, W_{0}\right)}{\varphi^{\prime}(\omega)}-\frac{\overline{B^{\prime}\left(\omega, W_{0}\right)}}{\varphi^{\prime}(\omega)}
$$

From (20) it is clear that for a fixed value of $a_{0}, a_{1}$ is a linear function of $a_{0}$ and $\bar{a}_{3}$ and can be written as

$$
a_{1}=\frac{a_{0}\left(\overline{B_{0}} B_{1}-A_{0} \overline{A_{1}}\right)+\bar{a}_{0}\left(B_{0} \overline{A_{1}}-\overline{A_{0}} B_{1}\right)}{\left|B_{1}\right|^{2}-\left|A_{1}\right|^{2}}+O\left(\rho^{2}\right),
$$

$$
\left|B_{1}\right|^{2} \neq\left|A_{1}\right|^{2},
$$


where we have put $A_{0}=A\left(\omega, W_{0}\right), A_{1}=A\left(\omega, W_{1}\right)$, and similarly for $B_{0}$ and $B_{1}$. We will show later that (24) can always be taken to be valid. Taking the case when (24) holds, we get on substituting this value of $a_{1}$ in (22) that

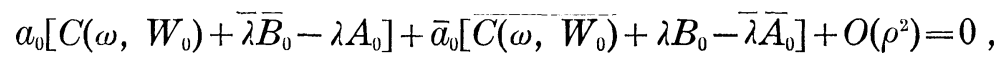

where

$$
\lambda=\frac{\overline{B_{1}} \overline{C\left(\omega, W_{1}\right)}+\overline{\mathrm{A}_{1}} C\left(\omega, W_{1}\right)}{\left|B_{1}\right|^{2}-\left|A_{1}\right|^{2}} .
$$

This holds for all sufficiently small values of $\rho$; hence because $a_{0}$ is arbitrary, in the limit $\rho \rightarrow 0$ the extremum function satisfies the equation

$$
C\left(\omega, W_{0}\right)=\lambda A\left(\omega, W_{0}\right)-\bar{\lambda} \overline{B\left(\omega, \overline{W_{0}}\right)},
$$

where $\lambda$ is independent of $W_{0}$. Again, because $W_{0}$ is an arbitrary point from (26), the equation satisfied by the extremum function can be written in the form

$$
\begin{aligned}
& \frac{(1+\alpha)(\omega-W)-\omega}{(\omega-W)^{2}}-\frac{\bar{\omega}(2+\bar{\alpha})(1-\bar{\omega} W)+\bar{\omega} W}{(1-\bar{\omega} W)^{2}}-\frac{\lambda \omega \varphi^{\prime}(\omega)}{\omega-W}+\frac{\overline{\lambda \omega^{2}} \varphi^{\prime}(\omega)}{1-\bar{\omega} W} \\
& \begin{array}{r}
W \varphi^{\prime 2}(W) \\
\varphi(W)
\end{array}\left[-\frac{\varphi(W)}{(\varphi(W)-\varphi(\omega))^{2}}-\frac{\varphi(\omega)(2-\varphi(\omega) \varphi(W))}{(1-\varphi(\omega) \varphi(W))^{2}}\right. \\
& \left.+\frac{\lambda \varphi(\omega)}{\varphi(W)-\varphi(\omega)}+\frac{\bar{\lambda} \overline{\varphi^{2}(\omega)}}{1-\varphi(\omega) \varphi(W)}\right],
\end{aligned}
$$

where

$$
\alpha=\omega \varphi^{\prime \prime}(\omega) / \varphi^{\prime}(\omega)=-f(\zeta) f^{\prime \prime}(\zeta)
$$

We now prove the following.

Lemma. For the extremum functions of the class $S$ which satisfy the equation (27), we have

$$
\mathfrak{\Im}\left\{1+\omega \varphi^{\prime \prime}(\omega) / \varphi^{\prime}(\omega)-\lambda \omega \varphi^{\prime}(\omega)\right\}=0
$$

and

$$
\Im(\lambda \varphi(\omega))=0
$$

Proof. Let us consider the variation 
(30)

$$
\begin{aligned}
& W^{*}=W+\frac{a_{0} \rho^{2} W}{W-W_{0}}-\frac{\bar{a}_{0} \rho^{2} W^{2}}{1-W_{0} W}+\frac{a_{1} \rho^{2} W}{W-W_{1}}-\begin{array}{c}
\bar{a}_{1} \rho^{2} W^{2} \\
1-W_{1} W
\end{array} \\
& +i T \rho^{2} W+O\left(\rho^{1}\right),
\end{aligned}
$$

where $T$ is real. It is easily seen that this variation keeps the origin and the unit circumference fixed and, for small enough $\rho$, is univalent on the boundary. So this is an acceptable variation. Under this variation the variation formula (17) will have the additional term $i T \rho^{2} W \varphi^{\prime}(W)$ on the right-hand side. This will give rise to an additional term $i T \mu^{2} \omega \varphi^{\prime}(\omega)$ in (19), and to $i T\left\{1+\omega \varphi^{\prime \prime}(\omega) / \varphi^{\prime}(\omega)\right\}$ in (21). Then, because (26) holds, the equation corresponding to (26) in this case will give rise to (28).

To prove (29) we observe that the derivation of the variational equation (17) leaves an arbitrariness which permits us to add a term $i k \varphi(W)$, for $k$ real, to the right hand side of (17). The addition of this term does affect the extremum condition (21), but it does appear as $i k \varphi(\omega)$ in the equation (20). The equation corresponding to (26) will then have an extra term $i k(\lambda \varphi(\omega))-\lambda \varphi(\omega)$, which must vanish since (26) has been proved to be the equation for the extremum function.

Transforming (27) in terms of $f(z)$ and using (28) and (29) we find that the extremum function statisfies the differential equation

$$
\begin{aligned}
& f^{\prime 2}(z)\left(f(z)-\alpha_{1}\right)\left(f(z)-\alpha_{2}\right) \\
& f(z)(f(z)-\omega)^{2}(1-\bar{\omega} f(z))^{2}
\end{aligned}=D \frac{\left(z-\beta_{1}\right)\left(z-\beta_{2}\right)}{z(z-\zeta)^{2}(1-\bar{\zeta} z)^{2}},
$$

where the constants $\alpha_{1}, \alpha_{2}, D, \beta_{1}$ and $\beta_{2}$ are obtained from (27) in the following form:

$$
\begin{aligned}
& D=\frac{\bar{\zeta}\left(-2|\zeta|^{2}+\lambda \zeta\left(|\zeta|^{2}-1\right)\right)}{\bar{\omega}\left(\beta\left(1-|\omega|^{2}\right)-\left(1+|\omega|^{2}\right)\right)}, \\
& f^{2}(z)+\frac{f(z) \frac{4|\omega|^{2}+\beta\left(-1+|\omega|^{2}\right)}{\bar{\omega}} \beta\left(1-|\omega|^{2}\right)-\left(1+|\omega|^{2}\right)}{\beta\left(\frac{\omega}{\bar{\omega}}\right.} \equiv\left(f(z)-\alpha_{1}\right)\left(f(z)-\alpha_{2}\right), \\
& z^{2}+\frac{z}{\zeta}-1+4|\zeta|^{2}+|\zeta|^{4}+\lambda \zeta\left(1-|\zeta|^{4}\right)+\frac{\zeta}{\zeta}=\left(z-\beta_{1}\right)\left(z-\beta_{2}\right),
\end{aligned}
$$

and

$$
\beta=1+\omega \varphi^{\prime \prime}(\omega) / \varphi^{\prime}(\omega)-\lambda \omega \varphi^{\prime}(\omega) .
$$

One further finds from (33) and (34) that $\left|\alpha_{1} \alpha_{2}\right|=1$ and $\left|\beta_{1} \beta_{2}\right|=1$. In order to fix $\lambda$ which remains arbitrary, as yet, we need the geometry of the extremum domain. In particular we prove the following.

THEOREM. If $f(z)$ is a function of the class $S$ for which $\left|f^{\prime}(\zeta)\right|$ is 
either a maximum or a minimum, then $f(z)$ maps the unit circle $|z|<1$ onto a slit domain.

Proof. If the theorem were not true, then there would exist a point $W_{0}, \quad\left|W_{0}\right|<1$ such that a neighborhood of $W_{0}$ is contained in $|W|<1$ and does not belong to the image domain. In the variation (16) taking $W_{0}$ and $W_{1}$ to be two such points we get the following variation formula for $f(z)$ :

$$
f^{*}(z)=f(z)+\rho^{2} \sum_{\nu=0}^{1}\left[\begin{array}{c}
a_{\nu} f(z) \\
f(z)-f\left(z_{\nu}\right)
\end{array}-\frac{\bar{a}_{\nu} f^{2}(z)}{1-f(z) f\left(z_{\nu}\right)}\right]+O\left(\rho^{+}\right) .
$$

The requirement that for all $f(z), f(\zeta)=f(\zeta)=\omega$, yields that

$$
\sum_{\nu=0}^{1}\left[\frac{a_{\nu}(1)}{\omega-f\left(z_{\nu}\right)}-\frac{\bar{a}_{\nu}\left(\omega^{2}\right.}{1-\omega f\left(z_{\nu}\right)}\right]+O\left(\rho^{2}\right)=0
$$

and the condition for the extremum of $\left|f^{\prime}(\zeta)\right|$ leads to

$$
\Re\left\{\sum_{\nu=0}^{1}\left[\frac{-a_{\nu} f\left(z_{\nu}\right)}{\left(\omega-f\left(z_{\nu}\right)\right)^{2}}-\frac{\bar{a}_{\nu}\left(2-\omega f\left(z_{\nu}\right)\right)}{\left(1-\omega f\left(z_{\nu}\right)\right)^{2}}\right]\right\}+O\left(\rho^{2}\right)=0 .
$$

Thus, because

$$
\left|\frac{1}{\omega-f\left(z_{\nu}\right)}\right|^{2}>\left|\begin{array}{c}
\omega \\
1-\omega f\left(z_{\nu}\right)
\end{array}\right|^{2}
$$

we see that the extremal function satisfies the equation

$$
\frac{f(z)}{(\omega-f(z))^{2}}+\frac{\bar{\omega}(2-\bar{\omega} f(z))}{(1-\bar{\omega} f(z))^{2}}=\frac{\lambda \omega}{\omega-f(z)}-\frac{\bar{\lambda} \bar{\omega}^{2}}{(1-\bar{\omega} f(z))^{2}} .
$$

But this is impossible since the left hand side has a second order pole at $z=\zeta$, where as the right hand side has only a first order pole.

As a consequence of this theorem it follows that (24) can always be assumed to hold. Indeed, if it were not so, we could find no point $z_{1}$ in $|z| \leqq 1$ such that $\left|A_{1}\right| \neq\left|B_{1}\right|$. Hence, because $A_{1}$ and $\bar{B}_{1}$ are analytic functions of $z_{1}$ and as equality is to hold for all $z_{1}$, we have

$$
A(\omega, f(z))=\mu \overline{B(\omega, f(z))}, \quad|\mu|=1 .
$$

(39) gives the following differential equation for $f(z)$ :

$$
\begin{aligned}
& f^{\prime 2}(z)\left[\left(\omega f^{\prime 2}(\zeta)-\mu \bar{\omega}^{2} f^{\prime}(\zeta)\right)-f(z)\left(|\omega|^{2} f^{\prime 2}(\zeta)-\mu \bar{\omega}^{2} f^{\prime}(\zeta)\right)\right] \\
& f^{\prime 2}(\zeta) f(\zeta) f(z)(f(z)-(1))(1-\bar{\omega} f(z)) \\
& =\frac{\zeta\left(1-\mu \overline{\zeta^{2}}\right)-z\left(|\zeta|^{2}-\mu \overline{\zeta^{2}}\right)}{z(z-\zeta)(1-\zeta z)} .
\end{aligned}
$$


But the function given by the differential equation (40) does not map the unit circle onto a slit domain, because at one end of the slit $f^{\prime}(z)$ will have a first order zero. Hence the right hand side of (40) should have a second order zero on the unit circumference. Since this is obviously not so, we have shown that (24) can always be taken to be valid.

We have thus shown that all extremal functions $f(z)$ which belong to $S$, and for which $\left|f^{\prime}(\zeta)\right|$ is a maximum or minimum, satisfy the differential equation (31). As the extremal function $f(z)$ maps $|z| \leqq 1$ onto a slit domain, at one end of the slit $f^{\prime}(z)$ will have a first order zero. To this zero of $f^{\prime}(z)$ there need to be a corresponding zero on the righthand side of (31), and as it is on the unit circumference $|z|=1$, we must have $\beta_{1}=\beta_{2}=e^{i r}$ in (31). Further, because the slit will make an angle $\theta$ with the unit circle such that $|\theta|<\pi$, we get from simple geometric considerations and the fact that the right hand side of (31) has no pole at any point on the unit circumference that $\alpha_{1}=\alpha_{2}=e^{i \theta}$. Geometrically this means that the slit starts from the unit circumference $|W|=1$, making an angle $\pi / 2$ with it.

As a result of the equality of $\alpha_{1}, \alpha_{2}$ and $\beta_{1}, \beta_{2}$, we have from (38) and (34) that

$$
f(\zeta) f^{\prime \prime}(\zeta)-\lambda \frac{f(\zeta)}{f^{\prime}(\zeta)}= \pm \frac{2|\omega|}{1-|\omega|^{2}}-1
$$

and

$$
i_{\zeta}=1 \mp \begin{gathered}
2|\zeta| \\
1-|\zeta|^{2}
\end{gathered}
$$

Eliminating $\lambda$ from both these equations one finds that, at the fixed point $\zeta$, the extremum function satisfies the equation

$$
\zeta f^{\prime \prime}(\zeta) f^{\prime}(\zeta)-\left(1 \mp \frac{2|\zeta|}{1-|\zeta|^{2}}\right)=\frac{\zeta f^{\prime}(\zeta)}{f(\zeta)}\left[-1 \pm \frac{2|f(\zeta)|}{1-|f(\zeta)|^{2}}\right]
$$

The differential equation (31) now reduces to

$$
\underset{f(z)(f(z)-\omega)^{2}(1-\bar{\omega} f(z))^{2}}{f^{\prime 2}(z)(f(z) \bar{\omega} \pm|\omega|)^{2}} \frac{(1 \mp|\omega|)^{2}}{\bar{\omega}}=\frac{(1 \mp|\zeta|)^{2}}{\zeta} \frac{(z \bar{\zeta} \mp|\zeta|)^{2}}{z(z-\zeta)^{2}(1-\zeta z)^{2}},
$$

where on each side either the upper or the lower sign is to be taken at one time.

From (44) one can get the information regarding the nature of the extremum domain. On account of the slit character of the extremum domain, the unit circumference $|W|=|f(z)|=1$ is definitely a part of the boundary. Further, if $z=e^{i \theta}$ we get from (44) that 


$$
\frac{z^{2} f^{\prime 2}(z)(f(z) \bar{\omega} \pm|\omega|)^{2}}{\left.f(z)(f(z)-(1))^{2}(1-\bar{\omega}) f(z)\right)^{2}} \frac{(1 \mp|\omega|)^{2}}{\bar{\omega}}
$$

is real. Hence, writing $W(t)=f(z)$ and making a proper choice of the parameter $t$, we can put it in the form

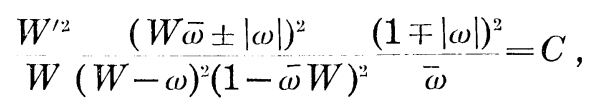

$C$ being some real constant. We now observe that this is an ordinary differential equation of the first order and hence has only one solution. Further, the straight line $W=r|c| \mid / \bar{\omega}$, where $r$ is a real parameter, does satisfy the differential equation. Since there is only one slit, this line corresponds to the slit and we conclude that the boundary of the image domain consists of the unit circumference and a radial slit pointing inwards at the points $\pm|\omega| \mid \bar{\omega}$.

Taking the square root and integrating (44) we obtain

$$
\begin{aligned}
& {\left[ \pm \log \frac{\sqrt{f}(z)-\sqrt{\omega}}{\sqrt{ } f(z)+\sqrt{\omega}}+\log \begin{array}{c}
1+\sqrt{\omega}{ }_{1-(z)} \\
1-\sqrt{\omega} \bar{f}(z)
\end{array}\right]+\text { const. }} \\
& = \pm\left[ \pm \log \frac{\sqrt{z}-\sqrt{\zeta}}{\sqrt{ } z-\sqrt{\zeta}}+\log \frac{1+\sqrt{\zeta} z}{1-\sqrt{\zeta} z}\right] .
\end{aligned}
$$

The various possibilities arising from different combinations of signs on the two sides of (46) are to be taken in such a way that the singularities at $f(z)=\omega$ and $z=\zeta$ on the two sides of (46) balance each other. We are thus left with only four possible combinations which after some simple algebra give rise to the following equations for the extremal functions:

$$
\frac{\left(1-|\omega|^{2}\right)(\omega . f(z)}{(\omega-|\omega| f(z))^{2}}=\frac{(1 \mp|\zeta|)^{2} z \zeta}{(\zeta \mp|\zeta| z)^{2}},
$$

and

$$
\begin{gathered}
(1+|\omega|)^{2} \omega f(z) \\
(\omega+|\omega| f(z))^{2}
\end{gathered}=\frac{(1 \pm|\zeta|)^{2} z \zeta}{(\zeta \pm|\zeta| z)^{2}}
$$

Equations (47) and (48), respectively, give rise to the following values of $f^{\prime}(\zeta)$ :

$$
f^{\prime}(\zeta)={ }_{\zeta}^{(1)} \frac{1 \pm|\zeta| 1-|\omega|}{1 \mp|\zeta| 1+|\omega|}
$$




$$
f^{\prime}(\zeta)={ }_{\zeta}^{\omega} 1 \mp|\zeta| \frac{1+|\omega|}{\zeta} 1 \pm|\zeta| \frac{1-|\omega|}{1+\mid}
$$

As $|\omega| \leqq|\zeta|$, one easily sees that the maximum value of $\left|f^{\prime}(\zeta)\right|$ is given by

$$
f^{\prime}(\zeta)=\frac{\omega}{\zeta} \frac{1+|\zeta|}{1-|\zeta|} \frac{1+|\omega|}{1-|\omega|}
$$

and that the function $f(z)$ corresponding to it is given by

$$
\frac{(1+|\omega|)^{2} \omega f(z)}{(\omega+|\omega| f(z))^{2}}=\frac{(1-|\zeta|)^{2} z \zeta}{(\zeta-|\zeta| z)^{2}}
$$

Also, the minimum of $\left|f^{\prime}(\zeta)\right|$ is given by

$$
f^{\prime}(\zeta)=\frac{\omega}{\zeta} \frac{1-|\zeta|}{1+|\zeta|} \frac{1-|\omega|}{1+|\omega|}
$$

and the function corresponding to it is given by

$$
\frac{(1-|\omega|)^{2} \omega . f(z)}{(\omega-|\omega| f(z))^{2}}=\frac{(1+|\zeta|)^{2} z \zeta}{(\zeta+|\zeta| z)^{2}} .
$$

We have thus proven the following.

THEOREM. Let $S$ denote the family of regular univalent functions $f(z)$ defined in the unit circle $|z| \leq 1$ such that $|f(z)| \leqq 1, f(0)=0$ and, for some fixed point $\zeta$ in $|z|<1, f(\zeta)=\omega$. Then the maximum and minimum values of $\left|f^{\prime}(\zeta)\right|$ are given by

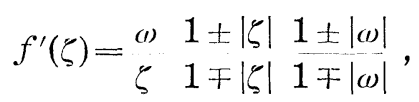

and the corresponding maximizing and minimizing functions are, respectively, given by the equation

$$
\frac{(1 \pm|\omega|)^{2} \omega f(z)}{(\omega \pm|\omega| f(z))^{2}}=\frac{(1 \mp|\zeta|)^{2} z \zeta}{(\zeta \mp|\zeta| z)^{2}}
$$

where the upper signs on both sides give the maximal function and the lower signs on both sides given the minimal function. The boundaries of the maximal and the minimal domains consist of the unit circumference together with radial slits starting, respectively, at the points $\pm|\omega| \mid \bar{\omega}$, the end points of the slits being the images of the points $\mp|\zeta| \bar{\zeta}$ by the corresponding functions given by (56). 
We now remark that we could as well have tried to solve the following problem:

In the class $S$ of regular univalent functions $f(z)$ in $|z| \leqq 1$ statisfying the normalization $f(0)=0,|f(z)| \leqq 1$ and, $f(\zeta)=\omega$ at some fixed point $\zeta$ in $|z|<1$, to find the function which maximizes $|f(\gamma)|$ at some $\eta \neq \zeta$ in $|z|<1$.

The existence and uniqueness of the solution is easily proven. It can also be readily shown that the extremal domain will be a slit damain. The variational equation for the extremum as obtained from (17) can be written as

$$
\begin{aligned}
\Re\left\{\left[\begin{array}{c}
a_{1} A\left(\omega_{1}, f\left(z_{0}\right)\right) \\
\omega_{1}
\end{array}-\bar{a}_{0} B\left(\omega_{1}, f\left(z_{1}\right)\right)\right.\right. & +\frac{a_{1} A\left(\omega_{1}, f\left(z_{1}\right)\right)}{\omega_{1}} \\
& \left.\left.-\frac{\bar{a}_{1} B\left(\omega_{1}, \bar{f}\left(z_{1}\right)\right)}{\omega_{1}}\right]\right\}=0,
\end{aligned}
$$

where $\omega_{1}=f(\eta)$, and we have replaced $\varphi(\omega)$ by $\zeta$ and $W_{0}$ by $f\left(z_{0}\right)$, $\varphi\left(W_{0}\right)=z_{0}, \varphi^{\prime}\left(W_{0}\right)$ by $1 / f^{\prime}\left(z_{0}\right)$, and similarly for $W_{1}$.

By arguments similar to those which lead to the equation (26) we can again assert that if

$$
C_{0}=\frac{A\left(\omega_{1}, f\left(z_{0}\right)\right)}{\omega_{1}}-\frac{A\left(\bar{\omega}_{1}, f\left(z_{0}\right)\right)}{\bar{\omega}_{1}},
$$

then the extremum function will satisfy the equation

$$
C_{0}=\mu A\left(\omega, f\left(z_{0}\right)\right)-\bar{\mu} \overline{B\left(\omega, f\left(z_{0}\right)\right)},
$$

where $\mu$ is independent of $f\left(z_{0}\right)$.

As in the lemma we can again show that $\mu f(\zeta) / f^{\prime}(\zeta)$ and $\mu \zeta$ are real. The differential equation for the extremum function can now be written as

$$
\begin{gathered}
f^{\prime 2}(z)\left(f(z)-c_{1}\right)\left(f(z)-d_{1}\right) \\
f(z)\left(f(z)-\omega_{1}\right)(f(z)-\omega)(1-\bar{\omega} f(z))\left(1-\bar{\omega}_{1} f(z)\right) \\
=K_{z(z-\eta)(z-\zeta)(1-\zeta z)(1-\bar{\eta} z)},
\end{gathered}
$$

where $\left|c_{1} d_{1}\right|=1$, and $\left|e_{1} e_{2}\right|=1$ and $c_{1}, d_{1}, e_{1}, e_{2}$ and $K$ can be determined by a comparison with (59).

From geometric considerations and the fact that the extremal domain will be a slight domain, one easily deduces that $c_{1}=d_{1}=e^{i \theta}$ and $e_{1}=e_{2}=e^{i \gamma}$. These conditions lead to 
(61)

$$
\left[\left(1-\left|\omega_{1}\right|^{2}\right)\left(1+|\omega|^{2}\right)-\mu_{1}\left(1-|\omega|^{2}\right)\left(1+\left|\omega_{1}\right|^{2}\right)\right]^{2}=4\left|\left(1-\left|\omega_{1}\right|^{2}\right) \omega-\mu_{1}\left(1-|\omega|^{2}\right) \omega_{1}\right|^{2},
$$

and

$$
\left[\left(1-|\eta|^{2}\right)\left(1+|\zeta|^{2}\right) \alpha_{1}-\mu \zeta\left(1-|\zeta|^{2}\right)\left(1+|\eta|^{2}\right)\right]^{2}=4\left|\left(1-|\eta|^{2}\right) \zeta \alpha_{1}-\mu \zeta \eta\left(1-|\zeta|^{2}\right)\right|^{2},
$$

where

$$
\mu_{1}=\mu f(\zeta) \mid f^{\prime}(\zeta)
$$

and

$$
\alpha_{1}=\eta f^{\prime}(\eta) / f(\eta) .
$$

Eliminating $\mu$ from (61) and (62), we get

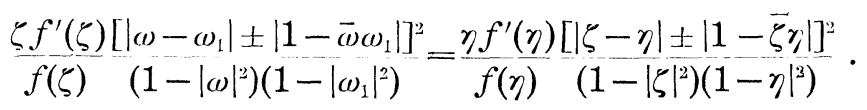

From the slit character of the extremal domain and geometric considerations we prove that the boundary of the extremal domain consists of the unit circumference with a slit that starts at right angles to the circumference. But we can no longer claim that this slit is radial. Also, because the integration of (60) involves hyperelliptic integrals it is not possible to get analytically any further information about the nature of the image domain. However, if one could show that the image domain is symmetric one could obtain an explicit result at least when $\zeta$ and $\eta$ are real. We are thus lead to reformulate the problem for bounded symmetric univalent functions.

4. Symmetrtc bounded univalent functions. We now want to construct a variation which keeps the unit circumference and the real axis fixed and which maps the origin into the origin. Evidently such a variation will be a combination of the variations considered in $\S \S 2$ and 3. One easily deduces that any such variation will be of the form

$$
W_{1}^{*}=W+\frac{a_{0} \rho^{2} W}{W-W_{0}}-\frac{\bar{a}_{0} \rho^{2} W^{2}}{1-W_{0} W}+\frac{\bar{a}_{0} \rho^{2} W}{W-\bar{W}_{0}}-\frac{a_{0} \rho^{2} W^{2}}{1-W_{0} W}+O\left(\rho^{1}\right)
$$

where $O\left(\rho^{4}\right)$ can be suitably chosen.

In order to be able to get a variation which can take account of some side conditions we need to take a linear combination of the variational terms in (64) with different $a_{0}$ and $W_{0}$. Thus, we get the variation

$$
W^{*}=W+\sum_{\nu=0}^{n}\left[\frac{a_{\nu} \rho^{2} W\left(1-W^{2}\right)}{\left(W-W_{\nu}\right)\left(1-W_{\nu} W\right)}+\frac{\bar{a}_{\nu} \rho^{2} W\left(1-W^{2}\right)}{\left(W-W_{\nu}\right)\left(1-W_{\nu} W\right)}\right]+O\left(\rho^{4}\right)
$$


The variation formula for $f(z)$ in this case is

(66)

$$
\begin{aligned}
f^{*}(z)= & f(z)+\rho^{2} \sum_{\nu=0}^{n}\left[\begin{array}{c}
a_{\nu} f(z)\left(1-f^{\prime \prime}(z)\right) \\
\left(f(z)-f\left(z_{\nu}\right)\right)\left(1-f(z) f\left(z_{\nu}\right)\right)
\end{array}\right. \\
& +\frac{\bar{a}_{\nu} f(z)\left(1-f^{\prime \prime}(z)\right)}{\left(f(z)-f\left(z_{\nu}\right)\right)\left(1-f(z) f\left(z_{\nu}\right)\right)}+\frac{a_{\nu} f^{\prime}(z) z\left(1-z^{2}\right)}{\left(z_{\nu}-z\right)\left(1-z_{\nu} z\right)} \frac{f\left(z_{\nu}\right)}{z_{\nu} f^{\prime \prime}\left(z_{\nu}\right)} \\
& \left.+\frac{\bar{a}_{\nu} f^{\prime}(z) z\left(1-z^{2}\right)}{\left(1-\bar{z}_{\nu} z\right)\left(\bar{z}_{\nu}-z\right) \overline{\bar{z}}_{\nu} f^{\prime \prime}\left(z_{\nu}\right)}\right]+O\left(\rho^{\prime}\right) .
\end{aligned}
$$

If we require $f^{*}(\zeta)=f(\zeta)=\omega$, $\zeta$ real, we get, using that $f(z)$ is symmetric,

$$
\Re\left[\sum a_{\nu}\left\{\frac{\omega\left(1-\omega^{2}\right)}{\left(\omega-f\left(z_{\nu}\right)\right)\left(1-\omega f\left(z_{\nu}\right)\right)}-\frac{\zeta\left(1-\zeta^{2}\right) f^{\prime}(\zeta)}{\left(\zeta-z_{\nu}\right)\left(1-\zeta z_{\nu}\right)} \frac{f\left(z_{\nu}\right)}{\left(z_{\nu} f^{\prime 2}\left(z_{\nu}\right)\right.}\right\}\right]+O\left(\mu^{2}\right)=0 .
$$

Also, if $\eta$ is real, then the condition for the extremum of $f(\eta)=\omega_{1}$ is

$$
\Re\left[a_{\nu}\left\{\frac{\omega_{1}\left(1-\omega_{1}^{2}\right)}{\left(\omega_{1}-f\left(z_{\nu}\right)\right)\left(1-\omega_{1} f\left(z_{\nu}\right)\right)}-\frac{\gamma_{1}\left(1-\eta^{2}\right) f^{\prime}(\eta)}{\left(\eta-z_{\nu}\right)\left(1-\eta z_{\nu}\right) z_{\nu} f^{\prime 2}\left(z_{\nu}\right)}\right\}\right]+O\left(\rho^{2}\right)=0 .
$$

Thus the extremum function satisfies the equations (67) and (68). By Lagrange's method of multipliers we see that the equation satisfied by the extremum function is

$$
\begin{array}{r}
z f^{\prime \prime 2}(z)\left[\frac{\left(1-\omega_{1}^{2}\right)}{f(z)}\left[\begin{array}{c}
\lambda\left(1-\omega^{2}\right) \\
\left(\omega_{1}-f(z)\right)\left(1-\omega_{1} f(z)\right)
\end{array}\right]\right. \\
=\frac{\alpha\left(1-\gamma^{2}\right)}{(\eta-z)\left(1-\gamma_{j} z\right)}-\frac{\alpha_{1} \lambda\left(1-\zeta^{\prime 2}\right)}{(\zeta-z)(1-\zeta z)},
\end{array}
$$

where

$$
\alpha=\eta f^{\prime}(\eta) / f(\eta)
$$

and

$$
\alpha_{1}=\zeta f^{\prime} /(\zeta)^{\prime} f(\zeta)
$$

It is easily proven in this case that the image domain is a slit domain. Thus, as in $\S 3$, from geometric considerations and the fact that the image domain is a slit domain we conclude that numerators on both the sides of (69) should be perfect squares. We thus have that either

$$
\alpha(1+\eta)(1-\zeta)=\alpha_{1} \lambda(1+\zeta)(1-\eta)
$$


or

$$
\alpha(1-\eta)(1+\zeta)=\alpha_{1} \lambda(1-\zeta)(1+\eta)
$$

and either

$$
\left(1+\omega_{1}\right)(1-\omega)=\lambda(1+\omega)\left(1-\omega_{1}\right)
$$

or

$$
\left(1-\omega_{1}\right)(1+\omega)=\lambda(1-\omega)\left(1+\omega_{1}\right) .
$$

The differential equation finally reduces to the form

$$
\begin{gathered}
\frac{\left(1 \pm \omega_{1}\right)\left(\omega-\omega_{1}\right)\left(1-\omega \omega_{1}\right) f^{\prime \prime 2}(z)(f(z) \mp 1)^{2}}{\left(1 \mp \omega_{l} f(z)\right)(\omega-f(z))\left(\omega_{1}-f(z)\right)(1-\omega f(z))\left(1-\omega_{1} f(z)\right)} \\
=\frac{\alpha(1 \pm \eta)(\zeta-\eta)(1-\zeta \eta)(z \mp 1)^{2}}{(1 \mp \eta) z(\zeta-z)(\eta-z)(1-\zeta z)(1-\eta z)},
\end{gathered}
$$

where the upper or the lower sign on each side is to be taken at one time.

The alternative in (70), (71) and (72), (73) arises on account of the ambiguity of sign of the root in (74).

In the left hand side of (74) let us make the transformation

$$
f(z)=\mp \begin{aligned}
& W+1 \\
& W-1
\end{aligned}
$$

according as we take the upper or the lower sign in (74). Then the left hand side transforms either to

$$
-\frac{16 W^{\prime 2} W^{2}\left(1+\omega_{1}\right)\left(\omega-\omega_{1}\right)\left(1-\omega \omega_{1}\right)}{(1+\omega)^{2}\left(1+\omega_{1}\right)^{2}\left(W^{2}-1\right)\left(W^{2}-\beta_{1}^{2}\right)\left(W^{2}-\beta_{2}^{2}\right)},
$$

or

$$
\frac{16 W^{\prime 2} W^{2}\left(1-\omega_{1}\right)\left(\omega-\omega_{1}\right)\left(1-\omega \omega_{1}\right)}{(1-\omega)^{2}\left(1-\omega_{1}\right)^{2}\left(W^{2}-1\right)\left(W^{2}-\beta_{1}^{-2}\right)\left(W^{2}-\beta_{2}^{-2}\right)},
$$

respectively, where $\beta_{1}=(1+\omega) /(1-\omega)$ and $\beta_{2}==\left(1+\omega_{1}\right) /\left(1-\omega_{1}\right)$.

Similarly, making the transformation

$$
z=\mp \begin{aligned}
& y+1 \\
& y-1
\end{aligned}
$$

according as we take the upper or the lower sign in the right hand side of (74), we get either 


$$
-\begin{array}{r}
16 \alpha y^{\prime 2} y^{2}(1+\eta)(1-\zeta \eta)(\zeta-\eta) \\
\left(y^{2}-1\right)\left(y^{2}-\gamma_{1}^{2}\right)\left(y^{2}-\gamma_{2}^{2}\right)(1-\eta)
\end{array},
$$

or

$$
-\frac{16 \alpha y^{\prime 2} y^{2}(1-\eta)(1-\zeta \eta)(\zeta-\eta)}{\left(y^{2}-1\right)\left(y^{2}-\gamma_{1}^{-2}\right)\left(y^{2}-\gamma_{2}^{-2}\right)(1+\eta)},
$$

respectively, where $\gamma_{1}=(1+\zeta) /(1-\zeta)$ and $\gamma_{2}=(1+\eta) /(1-\eta)$. We note that (77) is obtained from (76) by changing the signs of $\omega$ and $\omega_{1}$, and similarly (80) is obtained from (79) by changing the signs of $\zeta$ and $\eta$. Thus it is enough to consider the cases

$$
c_{1} \frac{W^{\prime} W}{V\left(W^{2}-1\right)\left(W^{2}-\beta_{1}^{2}\right)\left(W^{2}-\beta_{2}^{2}\right)}= \pm c_{2} \frac{y^{\prime} y}{\sqrt{\left(y^{2}-1\right)\left(y^{2}-\gamma_{1}^{2}\right)\left(y^{2}-\gamma_{2}^{2}\right)}},
$$

where

$$
c_{2}=\left[\begin{array}{c}
\alpha(1+\eta)(\zeta-\eta)(1-\zeta \eta) \\
(1-\eta)(1+\zeta)^{2}(1+\eta)^{2}
\end{array}\right]^{1 / 2}
$$

and

$$
c_{1}=\left[\begin{array}{c}
\left(1+\omega_{1}\right)\left(\omega-\omega_{1}\right)\left(1-\omega \omega_{1}\right) \\
(1+\omega)^{2}\left(1+\omega_{1}\right)^{2}
\end{array}\right]^{1 / 2} .
$$

Putting

$$
W_{2}=W^{2}-\frac{1}{3}\left(1+\beta_{1}^{2}+\beta_{2}^{2}\right),
$$

and

$$
X=y^{2}-\frac{1}{3}\left(1+\gamma_{1}^{2}+\gamma_{2}^{2}\right),
$$

we have from (81), on integration,

$$
c_{1} v=c_{2} u+\text { const. , }
$$

where $p(v)=W_{2}$ and $p^{*}(u)=X, p$ and $p^{*}$ being the Weierstrass's $p$ functions.

Since $f(0)=0, f(\zeta)=\omega$ and $f(\eta)=\omega_{1}$, we get, using the periodicity and homogeneity property of the $p$-functions,

$$
W_{2}=\frac{c_{1}^{2}}{c_{2}^{2}} X \text {. }
$$

Transforming back to $z$ and $f(z)$, we can write (82) in the form 
(83)

$$
\begin{aligned}
& {\left[\begin{array}{l}
f(z)-1 \\
f(z)+1
\end{array}\right]^{2}-\frac{1}{3}\left\{1+\left(\begin{array}{c}
1-(1) \\
1+(1)
\end{array}\right)^{2}+\left(\begin{array}{c}
1-(1)_{1} \\
1+(1)_{1}
\end{array}\right)^{2}\right\}} \\
& \quad=\frac{c_{1}^{2}}{c_{2}^{2}}\left[\left(\begin{array}{l}
z-1 \\
z+1
\end{array}\right)^{2}-\frac{1}{3}\left\{1+\left(\begin{array}{l}
1-\zeta \\
1+\zeta
\end{array}\right)^{2}+\left(\begin{array}{l}
1-\eta \\
1+\eta
\end{array}\right)^{2}\right\}\right] .
\end{aligned}
$$

Since $f(0)=0$ we have from (83) that

(84) $1-\frac{1}{3}\left\{1+\left(\frac{1-\omega}{1+\omega}\right)^{2}+\left(\frac{1-\omega_{1}}{1+\omega_{1}}\right)^{2}\right\}=\frac{c_{1}^{2}}{c_{2}^{2}}\left[1-\frac{1}{3}\left\{1+\left(\begin{array}{c}1-\zeta \\ 1+\zeta\end{array}\right)^{2}+\left(\frac{1-\eta}{1+\gamma}\right)^{2}\right\}\right]$.

This gives us $\omega_{1}$, but it involves $\alpha$ which is not yet known in terms of $\zeta, \omega$ and $\eta$. Towards this we observe that on subtracting (84) from (83) we have

$$
\frac{4 f(z)}{(f(z)+1)^{2}}=\frac{c_{1}^{2}}{c_{2}^{2}(1+z)^{2}},
$$

and because $f(\zeta)=\omega$ we have

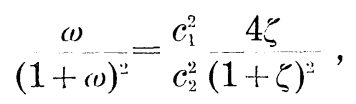

and finally

$$
\frac{f(z)(1+\omega)^{2}}{\omega(1+f(z))^{2}}=\frac{z(1+\zeta)^{2}}{(1+z)^{2} \zeta}
$$

Now, putting $z=\eta$ and $f(\eta)=\omega_{1}$, we get for $\omega_{1}$ the required equation

$$
\frac{(1+\omega)^{2}\left(\omega_{1}\right.}{\omega\left(1+\omega_{1}\right)^{2}}=\frac{\eta}{(1+\eta)^{2}}\left(\begin{array}{c}
(1+\zeta)^{2} \\
\zeta
\end{array}\right.
$$

Observing that all the possible extremal functions could be obtained by changing the signs of $\omega, \omega_{1}, \zeta$ and $\eta$ and taking all the combinations, we see that

$$
\frac{(1 \pm \omega)^{2}}{\omega} \frac{f(z)}{(1 \pm f(z))^{2}}= \pm \frac{z}{(1 \pm z)^{2}} \frac{(1 \pm \zeta)^{2}}{\zeta}
$$

gives all the extremal functions and

$$
\frac{(1 \pm \omega)^{2}\left(\omega_{1}\right.}{\omega\left(1 \pm\left(\omega_{1}\right)^{2}\right.}= \pm \frac{\eta}{(1 \pm \eta)^{2}} \quad(1 \pm \zeta)^{2}
$$

the corresponding values of $\omega_{1}$, where at one time either the upper or the lower sign is to be taken on each side of (88) and (89).

Further, on account of the continuity and univalence of $f(z)$ on the 
real axis, $f(z)$ will have the same sign or different sign as $z$ according as $\omega$ has the same or different sign as $\zeta$. Thus (88) and (89), respectively, reduce to

$$
\frac{(1 \pm \omega)^{2}}{\omega \quad(1 \pm f(z))^{2}}=\frac{z}{(1 \pm z)^{2}} \underset{\zeta}{(1 \pm \zeta)^{2}}
$$

and

$$
\frac{(1 \pm \omega)^{2}}{(1)} \frac{\omega_{1}}{\left(1 \pm \omega_{1}\right)^{2}}=\begin{gathered}
\eta \quad(1 \pm \zeta)^{2} \\
(1 \pm \eta)^{2}
\end{gathered}
$$

The following different cases need to be considered: (i) $\omega>0$, $\zeta>0$ and $\eta>0$ (ii) $\omega>0, \zeta>0$ and $\eta<0$ (iii) $\omega>0, \zeta<0$ and $\eta>0$ and (iv) $\omega>0, \zeta<0$ and $\eta<0$. We observe that (ii) can be easily deduced from (i) for in this case $\omega_{1}<0$, and the maximum and the minimum of $\omega_{1}$ in this case will be the same as the minimum and maximum of $\omega_{1}$ in (i). A similar relationship exists between (iii) and (iv). So we need to consider only the two cases (i) and (iii).

We now observe that if $|x|<1$ and $x$ is real then $x+1 / x$ is a monotonic decreasing function of $x$, and also that

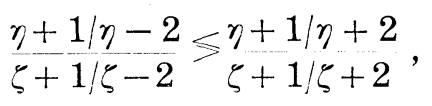

according as $\eta \gtrless \zeta>0$.

With these considerations we can prove the following.

Theorem. Let $\Sigma$ be the class of bounded, symmetric univalent functions $f(z)$ which are normalized so that $|f(z)| \leqq 1,|z| \leqq 1, f(0)=0$ and $f(\zeta)=\omega$ where $\zeta$ is a fixed real point in $|z|<1$. Further let $\omega>0$, $\zeta>0$ and for some real point $\eta$ let $f(\eta)=\omega_{1}$. Then the maximum and the minimum values of $\omega_{1}$, when $\eta>\zeta$, are given by

$$
\begin{array}{cc}
\omega_{1}+1 / \omega_{1} \pm 2 \\
\omega+1 / \omega \pm 2
\end{array} \begin{gathered}
\eta+1 / \eta \mp 2 \\
\zeta+1 / \zeta \mp 2
\end{gathered},
$$

and the corresponding maximizing and minimizing functions are respectively given by

$$
\underset{\omega}{(1 \pm \omega)^{2}} \frac{f(z)}{(1 \pm f(z))^{2}}=\underset{(1+z)^{2}}{z} \zeta^{2}
$$

where the upper signs on both sides give the maximum and the lower signs the minimum. However, if $\eta<\zeta$ then the maximum and minimum values given by (93) and the corresponding function given by (94) are interchanged. 
If $\omega<0, \zeta<0$ and $\eta>0$ then the maximum and the minimum values of $\omega_{1}$ are given by

$$
\frac{\omega_{1}+1 / \omega_{1} \pm 2}{\omega+1 / \omega \pm 2}=\frac{\eta+1 / \eta \pm 2}{\zeta+1 / \zeta \pm 2}
$$

and the corresponding maximizing and minimizing functions are given by

$$
\frac{(1 \pm \omega)^{2}}{\omega} \frac{f(z)}{(1 \pm f(z))^{2}}=\frac{z}{(1 \pm z)^{2}} \frac{(1 \pm \zeta)^{2}}{\zeta}
$$

where, as before, the upper sign on both sides gives the maximum and the lower sign gives the minimum.

The boundary of the extremal domain in each case consists of the unit circumference with a radial slit starting either at $W=1$ or $W=-1$. The length of the slit differs in various cases.

My thanks are due to Professor M. Schiffer for his interest and help in the progress and completion of the paper.

\section{REFERENCES}

1. M. Schiffer, A method of variation within the family of simple functions, Proc. London Math. Soc. (2), 44 (1938), 430-449.

2. - Variation of Green's function and theory of p-valued functions, Amer. J. Math. 65 (1943), 341-360.

3. - Applications of variational methods in the theory of conformal mapping (To be published in The Proceedings of the Symposia in Applied Mathematics 1956).

STANFORD UNIVERSITY 


\title{
PACIFIC JOURNAL OF MATHEMATICS
}

\author{
EDITORS
}

H. L. Royden

Stanford University

Stanford, California

R. A. Beaumont

University of Washington

Seattle 5, Washington
A. L. Whiteman

University of Southern California

Los Angeles 7, California

E. G. Straus

Unıversity of California

Los Angeles 24, California

\section{ASSOCIATE EDITORS}

\author{
E. F. BECKENBACH \\ C. E. BURGESS \\ M. HALL \\ E. HEWITT
}

\author{
A. HORN \\ V. GANAPATHY IYER \\ R. D. JAMES \\ M. S. KNEBELMAN
}

L. NACHBIN
I. NIVEN
T. G. OSTROM
M. M. SCHIFFER

G. SZEKERES

F. WOLF

K. YOSIDA

\section{SUPPORTING INSTITUTIONS}

UNIVERSITY OF BRITISH COLUMBIA

CALIFORNIA INSTITUTE OF TECHNOLOGY

UNIVERSITY OF CALIFORNIA

MON'TANA STATE UNIVERSITY

UNIVERSITY OF NEVADA

OREGON STATE COLLEGE

UNIVERSITY OF OREGON

UNIVERSITY OF SOUTHERN CALIFORNIA

\author{
STANFORD UNIVERSITY \\ UNIVERSITY OF UTAH \\ WASHINGTON STATE COLLEGE \\ UNIVERSITY OF WASHINGTON \\ AMERICAN MATHEMATICAL SOCIETY \\ CALIFORNIA RESEARCH CORPORATION \\ HUGHES AIRCRAFT COMPANY \\ THE RAMO-WOOLDRIDGE CORPORATION
}

Mathematical papers intended for publication in the Pacific Journal of Mathematics should be typewritten (double spaced), and the author should keep a complete copy. Manuscripts may be sent to any of the editors. All other communications to the editors should be addressed to the managing editor, E. G. Straus at the University of California, Los Angeles 24, California.

50 reprints per author of each article are furnished free of charge; additional copies may be obtained at cost in multiples of 50 .

The Pacific Journal of Mathematics is published quarterly, in March, June, September, and December. The price per volume (4 numbers) is $\$ 12.00$; single issues, $\$ 3.50$. Back numbers are available. Special price to individual faculty members of supporting institutions and to individual members of the American Mathematical Society: $\$ 4.00$ per volume; single issues, $\$ 1.25$.

Subscriptions, orders for back numbers, and changes of address should be sent to Pacific Journal of Mathematics, 2120 Oxford Street, Berkeley 4, California.

Printed at Kokusai Bunken Insatsusha (International Academic Printing Co., Ltd.), No. 10, 1-chome, Fujimi-cho, Chiyoda-ku, Tokyo, Japan.

PUBLISHED BY PACIFIC JOURNAL OF MATHEMATICS, A NON-PROFIT CORPORATION

The Supporting Institutions listed above contribute to the cost of publication of this Journal, but they are not owners or publishers and have no responsibility for its content or policies. 


\section{Pacific Journal of Mathematics}

\section{Vol. 7, No. $3 \quad$ March, 1957}

Silvio Aurora, Multiplicative norms for metric rings............... 1279

Ross A. Beaumont and John Richard Byrne, On the construction of

$R$-modules and rings with polynomial multiplication ............ 1305

Fred Brafman, An ultraspherical generating function . . . . . . . . . . . 1319

Howard Ernest Campbell, On the Casimir operator ............... 1325

Robert E. Edwards, Representation theorems for certain functional

operators..................................... 1333

Tomlinson Fort, The five-point difference equation with periodic

coefficients ..................................... 1341

Isidore Heller, On linear systems with integral valued solutions......... 1351

Harry Hochstadt, Addition theorems for solutions of the wave equation in

parabolic coordinates ................................ 1365

James A. Hummel, The coefficient regions of starlike functions . . . . . . . . 1381

Fulton Koehler, Estimates for the eigenvalues of infinite matrices ......... 1391

Henry Paul Kramer, Perturbation of differential operators ............. 1405

R. Sherman Lehman, Development of the mapping function at an analytic

corner .......................................... 1437

Harold Willis Milnes, Convexity of Orlicz spaces.................. 1451

Vikramaditya Singh, Interior variations and some extremal problems for certain classes of univalent functions . . . . . . . . . . . . . . . . 1485

William Lee Stamey, On generalized euclidean and non-euclidean spaces ............................................. 1505

Alexander Doniphan Wallace, Retractions in semigroups .............. 1513

R. L. Wilder, Monotone mappings of manifolds .................. 1519 